\title{
Influência dos indicadores de produção da cultura amendoim e suas relações na organização e agrupamento dos estados brasileiros
}

\author{
Submetido - 29 jul. $2020 \quad$ Aprovado - 20 set. $2020 \quad$ Publicado - 30 set. 2020
}

http://dx.doi.org/10.17648/sas.v1i149

\begin{abstract}
Paulo Alexandre da Silva (D)
Universidade Estadual Paulista "Júlio de Mesquita Filho", UNESP, Jaboticabal, São Paulo. E-mail: paullo-alex@outlook.com.
\end{abstract}

Maria Elisa Vicentini ${ }^{D}$

Universidade Estadual Paulista "Júlio de Mesquita Filho", UNESP, Jaboticabal, São Paulo. E-mail: mevicentini@gmail.com

Bruna Cristina de Lima Candido (D)

${ }^{3}$ Universidade Estadual Paulista "Júlio de Mesquita Filho", UNESP, Jaboticabal, São Paulo. E-mail: bruna_lcandido@hotmail.com.

Alan Rodrigo Panosso iD

${ }^{4}$ Universidade Estadual Paulista "Júlio de Mesquita Filho", UNESP, Jaboticabal, São Paulo. E-mail: alan.panosso@unesp.br.

Glauco de Sousa Rolim (D)

${ }^{5}$ Universidade Estadual Paulista "Júlio de Mesquita Filho", UNESP, Jaboticabal, São Paulo. E-mail: gasunesp.forms@gmail.com.

\section{RESUMO}

O amendoim é uma das principais culturas oleaginosas do mundo, apresentando diversos produtos derivados de seus grãos, como óleo, proteínas e vitaminas. Objetivo do trabalho foi organizar os estados brasileiros, em função dos indicadores de produção. As safras de 2019 e 2020 foram comparadas por meio da análise exploratória da técnica multivariada Cluster Analysis, pelo método hierárquico. Foram consideradas as variáveis de produção, produtividade e área colhida, para as safras de 2019 e 2020 no Brasil e em seus respectivos estados. Para a formação dos grupos foi usado à técnica multivariada exploratória de análise de agrupamentos, pelo método hierárquico. De maneira geral, os grupos foram separados em relação ao aumento, constância ou diminuição desses indicadores de produção, formando os seguintes grupos: Grupo 1 = Tocantins (TO); Grupo 2 = Alagoas (AL); Grupo 3 = São Paulo (SP), Mato Grosso do Sul (MS), Paraná (PR), Sergipe (SE), Ceará, (CE), Piauí (PI); Grupo 4 = Pará (PA), Maranhão (MA), Pernambuco (PE), Bahia (BA), Minas Gerais (MG), Mato Grosso (MT), Goiás (GO); Grupo 5 = Rondônia (RO), Acre (AC), Santa Catarina (SC), Rio Grande do Sul (RS). No Brasil existiu uma grande dispersão com relação aos indicadores de produção e o estudo multivariado pode ser de grande importância para os produtores, pois pode auxiliar nas tomadas de decisões sobre investimentos e colaborar com o entendimento sobre o avanço ou retrocesso da cultura do amendoim no território brasileiro.

Palavras-chave: Arachishypogaea L.; Brasil; Cluster analysis; Produtividade; Multivariada.

Influence of peanut culture production indicators and their relationships in the organization and grouping of brazilian states

\section{ABSTRACT}


Peanuts are one of the main oil crops in the world, presenting several products derived from their grains, such as oil, proteins and vitamins. The objective of the work was to organize the Brazilian states, according to the production indicators. The 2019 and 2020 harvests were compared using the exploratory analysis of the multivariate Cluster Analysis technique, using the hierarchical method. The variables of production, productivity and harvested area were considered for the 2019 and 2020 harvests in Brazil and in their respective states. For the formation of groups, the exploratory multivariate cluster analysis technique was used, using the hierarchical method. In general, the groups were separated in relation to the increase, constancy or decrease of these production indicators, forming the following groups: Group $1=$ Tocantins (TO); Group 2 = Alagoas (AL); Group 3 = São Paulo (SP), Mato Grosso do Sul (MS), Paraná (PR), Sergipe (SE), Ceará, (CE), Piauí (PI); Group 4 = Pará (PA), Maranhão (MA), Pernambuco (PE), Bahia (BA), Minas Gerais (MG), Mato Grosso (MT), Goiás (GO); Group 5 = Rondônia (RO), Acre (AC), Santa Catarina (SC), Rio Grande do Sul (RS). In Brazil there was a great dispersion in relation to the production indicators and the multivariate study can be of great importance for producers, as it can assist in making investment decisions and collaborate with the understanding of the advance or setback of the peanut culture in the territory Brazilian.

Keywords: Arachishypogaea L.; Brazil; Cluster analysis; Productivity, Multivariate.

\title{
Influencia de los indicadores de producción del cultivo de maní y sus relaciones en la organización y agrupación de los estados brasileños
}

\author{
RESUMEN
}

\begin{abstract}
El maní es uno de los principales cultivos oleaginosos del mundo, presentando varios productos derivados de sus granos, como aceite, proteínas y vitaminas. El objetivo del trabajo fue organizar los estados brasileños, de acuerdo con los indicadores de producción. Las cosechas de 2019 y 2020 se compararon mediante el análisis exploratorio de la técnica de Análisis de Conglomerados multivariante, utilizando el método jerárquico. Las variables de producción, productividad y área cosechada fueron consideradas para las cosechas 2019 y 2020 en Brasil y en sus respectivos estados. Para la formación de grupos se utilizó la técnica exploratoria de análisis de conglomerados multivariante, utilizando el método jerárquico. En general, los grupos se separaron en relación al aumento, constancia o disminución de estos indicadores de producción, conformando los siguientes grupos: Grupo 1 = Tocantins (TO); Grupo 2 = Alagoas (AL); Grupo 3 = São Paulo (SP), Mato Grosso do Sul (MS), Paraná (PR), Sergipe (SE), Ceará, (CE), Piauí (PI); Grupo 4 = Pará (PA), Maranhão (MA), Pernambuco (PE), Bahía (BA), Minas Gerais (MG), Mato Grosso (MT), Goiás (GO); Grupo 5 = Rondônia (RO), Acre (AC), Santa Catarina (SC), Rio Grande do Sul (RS). En Brasil hubo una gran dispersión en relación a los indicadores de producción y el estudio multivariado puede ser de gran importancia para los productores, ya que puede ayudar en la toma de decisiones de inversión y colaborar con la comprensión del avance o retroceso del cultivo del maní en el territorio. Brasileño.
\end{abstract}

Palabras clave: Arachishypogaea L.; Brasil, Análisis de conglomerados; Productividad; Multivariante.

\section{Introdução}

O amendoim (Arachishypogaea L.), pertence à família Fabaceae, é uma das leguminosas mais cultivadas no mundo. Originária do continente sul-americano pode ser cultivada em praticamente todos os tipos de solo. $\mathrm{O}$ Brasil ocupa a $12^{a}$ posição no ranking mundial de países produtores de 
amendoim, todavia na América latina o país ocupa o segundo lugar na produção e exportação, sendo inferior somente a Argentina (FAO, 2011).

Segundo Companhia Nacional de Abastecimento (CONAB, 2020), na safra 2019/2020, a área plantada com a cultura, teve um aumento $7,2 \%$ com uma produção de 516.500 toneladas. O seu cultivo está presente em todas as regiões do país, porém, segundo informações do Instituto de Economia Agrícola (IEA), descrita por Sampaio (2019), o Estado de São Paulo, representa $90 \%$ da produção brasileira, sendo o principal exportador. Contudo, conhecer o mercado interno em relação à produção e produtividade desse grão nos diferentes Estados, torna-se importante para visualizar o potencial econômico da cultura, estratégias de mercado e tomadas de decisões e possíveis expansões agrícolas do grão.

Com objetivo de identificar os grupos com produções ou características similares o uso de técnica multivariada com algoritmos de agrupamentos (análise de clusters) tem sido proposto diferentes áreas do agronegócio (MAJUMDAR et al. 2017). Portanto, o presente estudo teve como objetivo organizar os estados e as regiões brasileiras em função dos indicadores de produtividade da cultura do amendoim para as safras de 2018/2019 e 2019/2020, por meio da análise exploratória da técnica multivariada Cluster Analysis, agrupamentos, pelo método hierárquico.

\section{Material e métodos}

Os indicadores de produção da cultura do amendoim foram referentes aos estados brasileiros, nas safras de 2019 e 2020. O Brasil é um país expansivo $\left(8.516 .000 \mathrm{~km}^{2}\right)$ localizado entre os paralelos $5^{\circ} 16^{\prime} 20^{\prime}$ ' ' e $33^{\circ} 45^{\prime} 03^{\prime} \mathrm{S}$ e os meridianos $34^{\circ} 47^{\prime} 30^{\prime}$ ' E $73^{\circ} 59^{\prime} 32^{\prime}$ 'W na América do Sul. Segundo a classificação climática de Köppen, $81,4 \%$ do território compreende clima tropical, 4,9\% clima semi-árido e 13,7\% clima subtropical (Alvares et al., 2013). Possui 26 estados, porém devido à importância para a variável analisada foram estudados 19, Estados: Tocantins (TO), Sergipe (SE), Rondônia (RO), Piauí (PI), Acre (AC), Mato Grosso do Sul (MS) ), Mato Grosso (MT), Alagoas (AL), Goiás (GO), Pará (PA), Rio Grande do Sul (RS), 
Paraná (PR), Santa Catarina (SC), Maranhão (MA), Minas Gerais (MG), Bahia (BA), Pernambuco ( PE), Ceará (CE), São Paulo (SP).
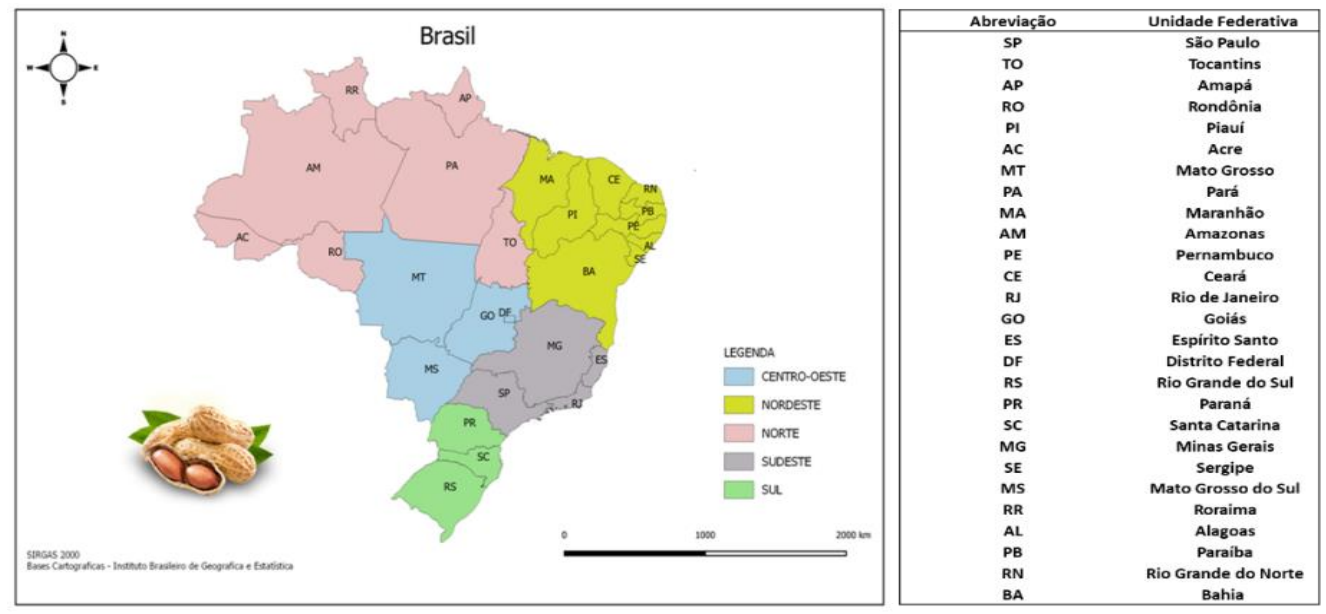

Figura 1. Distribuição geográfica dos estados brasileiros.

Os dados foram analisados, por meio da estatística descritiva (média, desvio-padrão, máximo, mínimo e coeficiente de variação). Foi realizado o teste de Teste de Shapiro-Wilk ao nível de $5 \%$ de probabilidade para verificação da normalidade dos dados. Em seguida foram aplicado o método estatístico multivariado, visando classificar os acessos em grupos: análise de agrupamentos hierárquica. Para essas análises foram consideradas variáveis de produção, produtividade e área colhida da cultura do amendoim nas safras de 2019 e 2020 para as regiões e estados brasileiros. Esses dados foram obtidos a partir da base do Instituto Brasileiro de Geografia e Estatística (IBGE), do Levantamento Sistemático da Produção Agrícola para área plantada, área colhida e produção, por ano da safra e produto das lavouras (IBGE, 2020).

\section{Resultados e discussão}

No estudo dos indicadores de produção foram observados que existiu uma grande dispersão quando comparados os estados brasileiros na safra de $2020 \mathrm{com}$ os de 2019. Para o Brasil os valores médios da área colhida, produção e produtividade apresentaram um aumento geral de 3.93, 18.84 e $15.62 \%$ (6736 hectares, 128630 toneladas, $618,66 \mathrm{~kg} \mathrm{ha}^{-1}$ ), respectivamente. Quando se compara entre os estados estudados observase uma diminuição de $54,74 \%$, sendo impulsionado principalmente pelo 
estado de TO em que diminuiu 1112,12 \% (400 para 33 hectares) e pelo máximo aumento que foi registrado no estado de MT $(41,86 \%$, que foi de 250 para 430 hectares) na safra de 2020, quando comparado com a de 2019 (Tabela 1).

Tabela 1. Estatísticas descritivas dos indicadores de área colhida, produção e produtividade da cultura de amendoim para todos os estados brasileiros estudados.

\begin{tabular}{cccccc}
\hline Indicadores de Produção & Média & DP & Mín & Máx & CV \\
\hline Área colhida (\%) & -54.74 & 256,75 & $-1112,12$ & 41,86 & 469,03 \\
Produção (\%) & -104.61 & 467,63 & $-2032,39$ & 41,86 & 447,03 \\
Produtividade (\%) & -3.99 & 30,58 & $-84,50$ & 37,08 & 765,88 \\
\hline DP = desvio padrão; Máx = Máximo; Mín = Mínimo; CV = coeficiente de variação (\%).
\end{tabular}

Com relação à produção foram observados os mesmos cenários, o TO foi o que mais diminuiu (2032,39\%, 1514 para 71 toneladas) e o MT foi o que mais aumentou (41.86\%, 1375 para 2365 toneladas). Já quando analisada a produtividade observa-se que o estado que mais aumentou quando comparamos a safra de $2020 \mathrm{com}$ a 2019 foi o SE (37.08 \%, 1600 para $2542.75 \mathrm{~kg} \mathrm{ha}^{-1}$ ), em contrapartida, o que mais diminuiu foi o $\mathrm{AL}$ com $84.50 \%$ (4691.49 para $\left.2542.82 \mathrm{~kg} \mathrm{ha}^{-1}\right)$.

Por meio da análise multivariada de agrupamentos foi gerado 0 dendrograma (Figura 2), o qual indicou a formação de 5 grupos em função da similaridade dos pontos amostrais, sendo os grupos: I = TO; II = AL; III = SE, MS, PR, SP, CE, PI; IV = MT, MG, GO, PE, BA, MA, PA; V = AC, RS, SC, RO.

De maneira geral, observa-se que os grupos III e IV estão apresentaram um crescimento na produção da cultura, devido ao aumento da produtividade, principalmente nos estados que formam o grupo III. Já os outros grupos (I, II e V) apresentaram a diminuição da produtividade, sendo o grupo o que mais diminuiu seguido do II e do V (Tabela 2). 


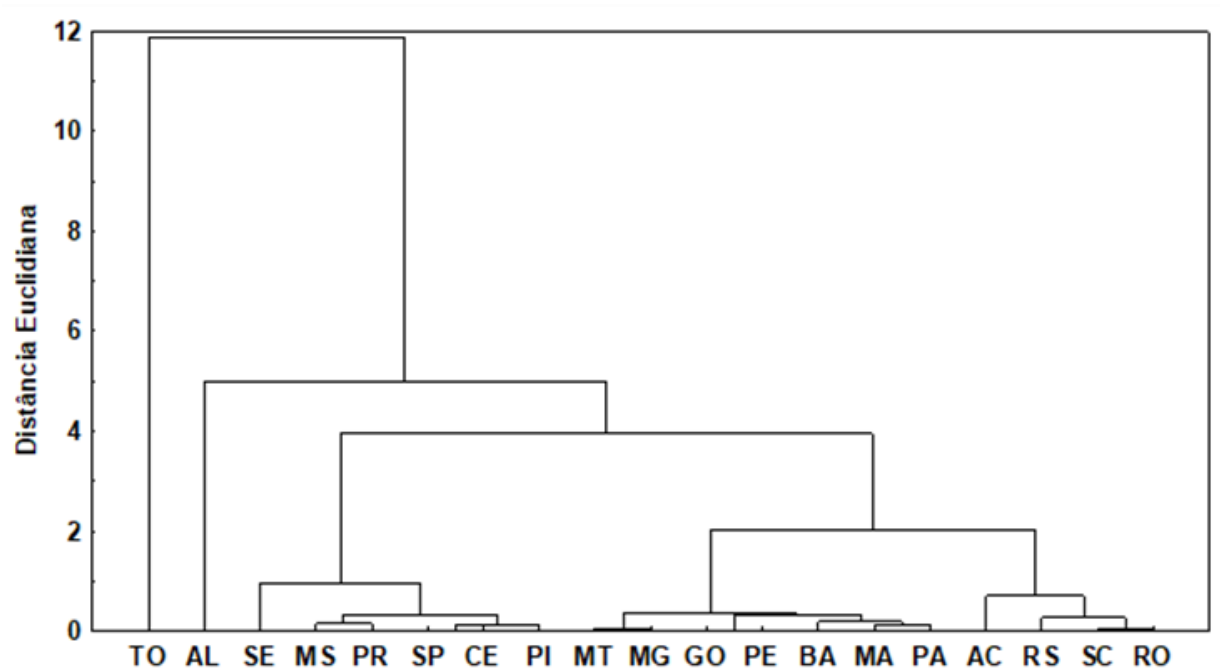

Figura 2. Dendrograma resultante da análise hierárquica de agrupamentos mostrando a formação de grupos segundo as porcentagens da área colhida, produção e produtividade da cultura do amendoim, na comparação entre a safra de 2019 e 2020 para os estados de TO, SE, RO, PI, AC, MS,MT, AL, GO, PA, RS, PR, SC, MA, MG, BA, PE, CE e SP.

Tabela 2. Estatísticas descritivas dos indicadores de área colhida, produção e produtividade da cultura de amendoim para todos os estados brasileiros estudados.

\begin{tabular}{|c|c|c|c|c|}
\hline Grupos & s Área colhida (\%) & Produção (\%) & Produtividade (\%) & Estados \\
\hline I & $\begin{array}{c}\text { Grande diminuição } \\
(1112.12)\end{array}$ & $\begin{array}{c}\text { Grande diminuição } \\
\text { (2032.39) }\end{array}$ & $\begin{array}{c}\text { Grande diminuição } \\
\text { (75.92) }\end{array}$ & TO \\
\hline II & $\begin{array}{c}\text { Grande aumento } \\
(30.11)\end{array}$ & $\begin{array}{c}\text { Grande diminuição } \\
(28.95)\end{array}$ & $\begin{array}{c}\text { Grande diminuição } \\
(84.50)\end{array}$ & AL \\
\hline III & $\begin{array}{l}\text { onstante ou pequena } \\
\text { diminuição (1.26) }\end{array}$ & Grande aumento (13.19) & Grande aumento (21.10) & $\begin{array}{l}\text { MS, PR, SP, } \\
\text { SE, CE, PI }\end{array}$ \\
\hline IV & $\begin{array}{c}\text { Grande aumento } \\
(14.56)\end{array}$ & Grande aumento (17.15) & $\begin{array}{l}\text { Constante ou pequeno } \\
\text { aumento (2.58) }\end{array}$ & $\begin{array}{l}\text { GO, MT, } \\
\text { MG, BA, PE, } \\
\text { MA, PA }\end{array}$ \\
\hline v & $\begin{array}{c}\text { Grande diminuição } \\
(13.11)\end{array}$ & $\begin{array}{c}\text { Grande diminuição } \\
(31.36)\end{array}$ & $\begin{array}{c}\text { Grande diminuição } \\
(15.03)\end{array}$ & $\begin{array}{c}\text { RO, AC, SC, } \\
\text { RS }\end{array}$ \\
\hline
\end{tabular}

\section{Conclusões}

A área colhida, a produção e a produtividade da cultura do amendoim no Brasil aumentaram. Nos indicadores entre os estados, foi observado grandes variações com relação ao aumento, constância e diminuição da porcentagem dos indicadores de produção, quando se comparada as safras de 2019/2020 e 2018/2019, permitindo organizar os estados brasileiros em 5 grupos, sendo estes: I = TO; II = AL; III = SE, MS, PR, SP, CE, PI; IV = MT, MG, GO, PE, BA, MA, PA; V = AC, RS, SC, RO. O estudo multivariado pode ser de grande importância para os produtores, pois 
pode auxiliar nas tomadas de decisões sobre investimentos e colabora com o entendimento sobre o avanço ou retrocesso da cultura do amendoim no território brasileiro.

\section{Agradecimentos}

Agrademos à Coordenação de Aperfeiçoamento de Pessoal de Nível Superior (CAPES). 


\section{Referências}

ALVARES, C. A., StAPE, J. L., SENTElHAS, P. C., DE MORAES, G., LEONARDO, J., \& SPAROVEK, G. Köppen's climate classification map for Brazil. Meteorologische Zeitschrift, v.22, n.6, p. 711-728, 2013. https://doi.org/10.1127/0941-2948/2013/0507

Companhia Nacional de Abastecimento - CONAB. (2020). Levantamento da safra 2019/2020: grãos. Brasília: Conab.

Food Agriculture Organization of United Nations (FAO). Disponível em: http://www.fao.org/corp/statistics. Acesso em: 02 de fevereiro. 2011.

Instituto Brasileiro de Geografia e Estatística - IBGE. (2020). Levantamento sistemático da produção agrícola. Tabela 188 - Rendimento médio, por ano da safra e produto das lavouras. IBGE. Disponível em: https://sidra.ibge.gov.br/tabela/1618. Acesso em: 27 julho. 2020.

MAJUMDAR, J., NARASEEYAPPA, S. AND ANKALAKI, S. Analysis of agriculture data using data mining techniques: application of big data. J Big Data, v. 4, 20p., 2017. https://doi.org/10.1186/s40537-017-007

SAMPAIO, R. M. Amendoim: exportações em alta e a importante participação dos municípios paulistas. Análise e Indicadores do Agronegócio, v. $14, \quad$ n. 4, p. 1-5, abr. 2019. Disponível em: http://www.iea.sp.gov.br/ ftpiea/AIA/AIA-23-2019. pdf. Acesso em: 27 julho. 2020. 